\title{
The Reproducing Kernel Hilbert Space Method for Solving System of Linear Weakly Singular Volterra Integral Equations
}

\author{
Hameeda O. AL-Humedi and Ameer S. Abdul-hasan
}

\begin{abstract}
Department of Mathematics, University of Basrah, College of Education for Pure Sciences, Basrah, Iraq. hameedao@yahoo.com, amrstrabd@gmail.com
\end{abstract}

\begin{abstract}
The exact solutions of a system of linear weakly singular Volterra integral equations (VIE) have been a difficult to find. The aim of this paper is to apply reproducing kernel Hilbert space (RKHS) method to find the approximate solutions to this type of systems. At first, we used Taylor's expansion to omit the singularity. From an expansion the given system of linear weakly singular VIE is transform into a system of linear ordinary differential equations (LODEs). The approximate solutions are represent in the form of series in the reproducing kernel space $W_{1}[0,1]$. By comparing with the exact solutions of two examples, we saw that RKHS is a powerful, easy to apply and full efficiency in scientific applications to build a solution without linearization and turbulence or discretization .
\end{abstract}

Keywords: Linear system, weakly singular kernel, Volterra integral equations, Taylor's expansion, reproducing kernel Hilbert space.

Mathematics Subject Classifications 2010 [MSC]: 76S05, 65N99, 35Q35.

\section{1- Introduction}

In this paper, we consider the following system of linear weakly singular VIE of the following form [1]:

$\left.\begin{array}{l}u_{1}(x)=f_{1}(x)+\int_{a}^{x}\left(k_{11}(x, y) u_{1}(y)+k_{12}(x, y) u_{2}(y)\right) d y, \\ \left.u_{2}(x)=f_{2}(x)+\int_{a}^{x}\left(k_{21}(x, y) u_{1}(y)+k_{22}(x, y) u_{2}(y)\right)\right) d y\end{array}\right\}, a \leq x \leq b$

where $a$ and $b$ are real finite constant and the functions, $u_{j}(x) \in W_{1}[0,1], j=1,2$ are unknown functions to be determined, $f_{j}(x), j=1,2$ are continuous functions or square integrable on $[\mathrm{a}, \mathrm{b}]$, and the functions $u_{j}(y)$, are

linear functions. The kernels $k_{j l}$ are singular kernels of the generalized from given by $k_{j l}=\frac{1}{[g(x)-g(y)]^{\alpha_{j l}}}, g(x)=$ $x, 1 \leq j, l \leq 2,0 \leq \alpha_{j l} \leq 1$. Several valid methods for solving VIE have been developed in recent years, including power series method[2], Adomain's decomposition method [3], homotopy perturbation method [4,5], block by block method [6], and expansion method [7].

Reproducing kernel theory has important application in numerical analysis, differential equation, probability and statistics and so on $[8,9]$, and the RKHS method to applied approximate method in the space $W_{1}[0,1]$

For obtaining the numerical solutions.

This paper organizing as follows: To transforming (1.1) into a system of LODEs using Taylor's expansion will be put forward in the section 2. In section 3, construction of RKHS. Discusses the implementation of method in section 4 . In section 5, the numerical examples are present. Finally, the conclusions will give in section 6 .

\section{2- Taylor's Expansion}

Consider the following system of linear weakly singular VIEs: 


$$
\left.\begin{array}{l}
u_{1}(x)=f_{1}(x)+\int_{a}^{x}\left(\frac{1}{[g(x)-g(y)]^{\alpha_{11}}} u_{1}(y)+\frac{1}{[g(x)-g(y)]^{\alpha_{12}}} u_{2}(y)\right) d y, \\
u_{2}(x)=f_{2}(x)+\int_{a}^{x}\left(\frac{1}{[g(x)-g(y)]^{\alpha_{21}}} u_{1}(y)+\frac{1}{[g(x)-g(y)]^{\alpha_{22}}} u_{2}(y)\right) d y
\end{array}\right\}
$$

rewriting (2.1) as follows:

$$
\left.\begin{array}{rl}
u_{1}(x) & =f_{1}(x)+\frac{(g(x)-g(a))^{1-\alpha_{11}} u_{1}(x)}{\left(1-\alpha_{11}\right) g^{\prime}(a)}-\int_{a}^{x}(g(x)-g(y))^{1-\alpha_{11}} \frac{u_{1}(y)-u_{1}(x)}{g(y)-g(x)} d y \\
& +\frac{(g(x)-g(a))^{1-\alpha_{12}} u_{2}(x)}{\left(1-\alpha_{12}\right) g^{\prime}(a)}-\int_{a}^{x}(g(x)-g(y))^{1-\alpha_{12}} \frac{u_{2}(y)-u_{2}(x)}{g(y)-g(x)} d y \\
u_{2}(x) & =f_{2}(x)+\frac{(g(x)-g(a))^{1-\alpha_{21}} u_{1}(x)}{\left(1-\alpha_{21}\right) g^{\prime}(a)}-\int_{a}^{x}(g(x)-g(y))^{1-\alpha_{21}} \frac{u_{1}(y)-u_{1}(x)}{g(y)-g(x)} d y \\
& +\frac{(g(x)-g(a))^{1-\alpha_{22}} u_{2}(x)}{\left(1-\alpha_{22}\right) g^{\prime}(a)}-\int_{a}^{x}(g(x)-g(y))^{1-\alpha_{22}} \frac{u_{2}(y)-u_{2}(x)}{g(y)-g(x)} d y
\end{array}\right\}
$$

by using Taylor's expansion about $y=x$ of degree $\mathrm{n}$ for $u_{1}(y)$ and $u_{2}(x)$ we get:

$$
\left.\begin{array}{l}
u_{1}(y)-u_{1}(x) \approx(g(y)-g(x)) u_{1}^{\prime}(x)+\frac{(g(y)-g(x))^{2}}{2 !} u_{1}^{\prime \prime}(x)+\cdots+\frac{(g(y)-g(x))^{n}}{n !} u_{1}^{(n)}(x) \\
u_{2}(y)-u_{2}(x) \approx(g(y)-g(x)) u_{2}^{\prime}(x)+\frac{(g(y)-g(x))^{2}}{2 !} u_{2}^{\prime \prime}(x)+\cdots+\frac{(g(y)-g(x))^{n}}{n !} u_{2}^{(n)}(x)
\end{array}\right\}
$$

substituting (2.3) into the right hand side of (2.2) respectively, yields

$$
\left.\begin{array}{l}
u_{1}(x)=f_{1}(x)+\sum_{i=0}^{n}(-1)^{i} \frac{(g(x)-g(a))^{i+1-\alpha_{11}}}{i !\left(i+1-\alpha_{11}\right) g^{\prime}(a)} u_{1}^{(i)}(x)+\sum_{i=0}^{n}(-1)^{i} \frac{(g(x)-g(a))^{i+1-\alpha_{12}}}{i !\left(i+1-\alpha_{12}\right) g^{\prime}(a)} u_{2}^{(i)}(x) \\
u_{2}(x)=f_{2}(x)+\sum_{i=0}^{n}(-1)^{i} \frac{(g(x)-g(a))^{i+1-\alpha_{21}}}{i !\left(i+1-\alpha_{21}\right) g^{\prime}(a)} u_{1}^{(i)}(x)+\sum_{i=0}^{n}(-1)^{i} \frac{(g(x)-g(a))^{i+1-\alpha_{22}}}{i !\left(i+1-\alpha_{22}\right) g^{\prime}(a)} u_{2}^{(i)}(x)
\end{array}\right\}
$$

suppose that

$$
\begin{aligned}
& N_{1}\left(x, u_{1}(x)\right)=\sum_{i=0}^{n}(-1)^{i} \frac{(g(x)-g(a))^{i+1-\alpha_{11}}}{i !\left(i+1-\alpha_{11}\right) g^{\prime}(a)} u_{1}^{(i)}(x), \quad N_{2}\left(x, u_{1}(x)\right)=\sum_{i=0}^{n}(-1)^{i} \frac{(g(x)-g(a))^{i+1-\alpha_{21}}}{i !\left(i+1-\alpha_{21}\right) g^{\prime}(a)} u_{1}^{(i)}(x) \\
& N_{2}\left(x, u_{1}(x)\right)=\sum_{i=0}^{n}(-1)^{i} \frac{(g(x)-g(a))^{i+1-\alpha_{21}}}{i !\left(i+1-\alpha_{21}\right) g^{\prime}(a)} u_{1}^{(i)}(x), \quad \widetilde{N}_{2}\left(x, u_{2}(x)\right)=\sum_{i=0}^{n}(-1)^{i} \frac{(g(x)-g(a))^{i+1-\alpha_{22}}}{i !\left(i+1-\alpha_{22}\right) g^{\prime}(a)} u_{2}^{(i)}(x)
\end{aligned}
$$

then (2.4) take the forms:

$$
\left.\begin{array}{l}
u_{1}(x)=f_{1}(x)+N_{1}\left(x, u_{1}(x)\right)+\widetilde{N}_{1}\left(x, u_{2}(x)\right) \\
u_{2}(x)=f_{2}(x)+N_{2}\left(x, u_{1}(x)\right)+\widetilde{N}_{2}\left(x, u_{2}(x)\right)
\end{array}\right\}
$$

respectively. Therefore, (2.1) can be approximate by the $n$ th-order system of LODEs (2.5).

\section{3- Construction of RKHS}

Definition 3.1 Let $\mathrm{H}$ be a Hilbert space of functions $u: X \rightarrow \mathbb{R}$. Denote by $\langle.,$.$\rangle the inner product and let$ $\|\|=.\sqrt{\langle.,\rangle}$ be the induced norm in $\mathrm{H}$ the function $R: X \times X \rightarrow \mathbb{R}$ is called a reproducing kernel of $\mathrm{H}$ if the followings are satisfied [10]

(i) $R_{x}()=.R(x,.) \in \mathrm{H}, \forall x \in X$,

(ii) $\left\langle u(),. R_{x}().\right\rangle=u(x), \forall u \in \mathrm{H}$, for all $x \in X$ (the reproducing property). 
The value of the function $u$ at the point $x$ is reproduce by the inner product of $u$ with $R(x,$.$) . Then \mathrm{H}$ called a RKHS.

\subsection{The reproducing kernel Hilbert space $W_{1}[0,1]$}

The inner product space $W_{1}[0,1]$ is defined as $W_{1}[0,1]=\left\{u_{1}(x) \mid u_{1}(x)\right.$ is an absolutely continuous real valued function in $\left.[0,1], u_{1}^{\prime}(x) \in L^{2}[0,1]\right\}$. The inner product and norm in $W_{1}[0,1]$ are given by [11],

$$
\begin{aligned}
&\left\langle u_{1}(x), u_{2}(x)\right\rangle_{W_{1}}=u_{1}(0) u_{2}(0)+\int_{0}^{1} u_{1}^{\prime}(x) u_{2}^{\prime}(x) d x, \quad u_{1}(x), u_{2}(x) \in W_{1}[0,1] \\
&\left\|u_{1}\right\|_{W_{1}}=\sqrt{\left\langle u_{1}(x), u_{1}(x)\right\rangle_{W_{1}}} \quad, \quad \text { where } \quad u_{1}(x) \in W_{1}[0,1]
\end{aligned}
$$

respectively. It has been prove that $W_{1}[0,1]$ is a complete reproducing kernel space.

Theorem 3.1 The reproducing kernel of $W_{1}[0,1]$ is

$$
R_{x}(y)= \begin{cases}1+y, & y \leq x \\ 1+x, & y>x\end{cases}
$$

Proof. Appling (3.1), we have

$$
\left\langle u_{1}(y), R_{x}(y)\right\rangle_{W_{1}}=u_{1}(0) R_{x}(0)+\int_{0}^{1} u_{1}^{\prime}(y) R_{x}^{\prime}(y) d y,
$$

by integrating (3.4) by parts gives

$$
\left\langle u_{1}(y), R_{x}(y)\right\rangle_{W_{1}}=u_{1}(0)\left(R_{x}(0)-R_{x}^{\prime}(0)\right)+u_{1}(1) R_{x}^{\prime}(1)-\int_{0}^{1} u_{1}(y) R_{x}^{\prime \prime}(y) d y
$$

in order to $\left\langle u_{1}(y), R_{x}(y)\right\rangle_{W_{1}}=u_{1}(x)$, it is enough to request the following equalities hold

$$
\begin{aligned}
-R_{x}^{\prime \prime}(y) & =\delta(y-x) \\
R_{x}(0) & =R_{x}^{\prime}(0), \quad R_{x}^{\prime}(1)=0,
\end{aligned}
$$

where $\delta$ is a Dirac delta function. From (3.5), if $y \neq x$ then $R_{x}^{\prime \prime}(y)=0$, have a characteristic equation is $\lambda^{2}=0$. Then we obtain

$$
R_{X}(y)= \begin{cases}a_{1}(x)+a_{2}(x) y, & y \leq x \\ b_{1}(x)+b_{2}(x) y, & y>x\end{cases}
$$

integrate both sides of (3.5) from $x-\varepsilon$ to $x+\epsilon$ with respect to $y$ and let $\varepsilon \rightarrow 0$, one gets

$$
\begin{aligned}
& R_{x}(x+0)=R_{x}(x-0), \\
& R_{x}^{\prime}(x-0)-R_{x}^{\prime}(x+0)=1,
\end{aligned}
$$

to obtain (3.3) must be solve (3.5), (3.6), (3.8) and (3.9) then substituting the results of $a_{1}, a_{2}, b_{1}$ and $b_{2}$ into (3.7).

\section{4- The Method Implementation}

To implement the RKHS method, we rewrite equations (2.5) are follows

$$
L u_{1}(x)=f_{1}(x)+N_{1}\left(x, u_{1}(x)\right)+\widetilde{N}_{1}\left(x, u_{2}(x)\right),
$$




$$
L u_{2}(x)=f_{2}(x)+N_{2}\left(x, u_{1}(x)\right)+\widetilde{N}_{2}\left(x, u_{2}(x)\right),
$$

where, $L: W_{1}[0,1] \rightarrow W_{1}[0,1]$ is an invertible bounded linear operator, $N_{1}, \widetilde{N}_{1}, N_{2}$ and $\widetilde{N}_{2} \in W_{1}[0,1]$ and $f_{1}(x), f_{2}(x)$ are continuous functions in $W_{1}[0,1] . W_{1}[0,1]$ is a reproducing kernel space defined according to the highest derivatives involved in (2.5). We choose a countable set of points $\left\{x_{m}\right\}_{m=1}^{\infty}$ in the interval $[0,1]$. Define

$$
\varphi_{m}(x)=R\left(x, x_{m}\right), \quad \psi_{m}(x)=L^{*} \varphi_{m}(x)
$$

where $L^{*}$ is the conjugate operator of $L$.

The orthonormal system of $\left\{\bar{\psi}_{m}\right\}_{m=1}^{\infty}$ from the space $W_{1}[0,1]$ can be derive from Gram-Schmidt orthogonal process of $\left\{\psi_{m}\right\}_{m=1}^{\infty}$,

$$
\bar{\psi}_{m}(x)=\sum_{k=1}^{m} \beta_{m k} \psi_{k}(x)
$$

where $\beta_{m k}$ are orthogonalization coefficients such that $\beta_{m m}>0, \quad m=1,2, \ldots$

Theorem 4.1 If $\left\{x_{m}\right\}_{m=1}^{\infty}$ is dense on $[0,1]$ then $\left\{\psi_{m}\right\}_{m=1}^{\infty}$ is the complete function system of the space $W_{1}[0,1]$ and $\psi_{m}(x)=\left.L_{y} R_{x}(y)\right|_{y=x_{m}}$, where the subscript $y$ in the operator $L$ indicates that the operator $L$ applies to the function of $y$ [12].

Proof. We have

$$
\begin{aligned}
\psi_{m}(x) & =\left\langle\psi_{m}(y), R_{x}(y)\right\rangle_{W_{1}[0,1]}=\left\langle\left(L^{*} \varphi_{m}\right)(y), R_{x}(y)\right\rangle_{W_{1}[0,1]} \\
& =\left\langle\varphi_{m}(y), L_{y} R_{x}(y)\right\rangle_{W_{1}[0,1]}=\left.L_{y} R_{x}(y)\right|_{y=x_{m}} .
\end{aligned}
$$

$\forall u(x) \in W_{1}[0,1]$, let $\left\langle u(x), \psi_{m}(x)\right\rangle_{W_{1}[0,1]}=0, \quad m=1,2, \ldots$

i.e. $\quad\left\langle u(x),\left(L^{*} \varphi_{m}\right)(x)\right\rangle_{W_{1}[0,1]}=\left\langle L u(.), \varphi_{m}(.)\right\rangle_{W_{1}[0,1]}$

$$
=L u\left(x_{m}\right)=0
$$

$L u(x)=0$ when $\left\{x_{m}\right\}_{m=1}^{\infty}$ is dense on $[0,1]$, if $L^{-1}$ exists then $u(x)=0$.

Theorem 4.2 If $\left\{x_{m}\right\}_{m=1}^{\infty}$ is dense on $[0,1]$ and $u_{1}(x), u_{2}(x) \in W_{1}[0,1]$ are the solutions of equations (4.1), then these solutions are satisfy the following form,

$$
\left.\begin{array}{l}
u_{1}(x)=\sum_{m=1}^{\infty} \sum_{k=1}^{m} \beta_{m k}\left(f_{1}(x)+N_{1}\left(x, u_{1}(x)\right)+\widetilde{N}_{1}\left(x, u_{2}(x)\right)\right) \bar{\psi}_{m}(x) \\
u_{2}(x)=\sum_{m=1}^{\infty} \sum_{k=1}^{m} \beta_{m k}\left(f_{2}(x)+N_{2}\left(x, u_{1}(x)\right)+\widetilde{N}_{2}\left(x, u_{2}(x)\right)\right) \bar{\psi}_{m}(x),
\end{array}\right\}
$$

respectively.

Proof. By definition (4.2), for $u_{1}(x)$ in (4.5) we have

$$
\begin{aligned}
u_{1}(x) & =\sum_{m=1}^{\infty}\left\langle u_{1}(x), \bar{\psi}_{m}(x)\right\rangle_{W_{1}[0,1]} \bar{\psi}_{m}(x) \\
& =\sum_{m=1}^{\infty}\left\langle u_{1}(x), \sum_{k=1}^{m} \beta_{m k} \psi_{k}(x)\right\rangle_{W_{1}[0,1]} \bar{\psi}_{m}(x) \\
& =\sum_{m=1}^{\infty} \sum_{k=1}^{m} \beta_{m k}\left\langle u_{1}(x), L^{*} \varphi_{k}(x)\right\rangle_{W_{1}[0,1]} \bar{\psi}_{m}(x) \\
& =\sum_{m=1}^{\infty} \sum_{k=1}^{m} \beta_{m k}\left\langle L u_{1}(x), \varphi_{k}(x)\right\rangle_{W_{1}[0,1]} \bar{\psi}_{m}(x)
\end{aligned}
$$




$$
\begin{aligned}
& =\sum_{m=1}^{\infty} \sum_{k=1}^{m} \beta_{m k} L u_{1}\left(x_{k}\right) \bar{\psi}_{m}(x) \\
& =\sum_{m=1}^{\infty} \sum_{k=1}^{m} \beta_{m k}\left(f_{1}\left(x_{k}\right)+N_{1}\left(x_{k}, u_{1}\left(x_{k}\right)\right)+\widetilde{N}_{1}\left(x_{k}, u_{2}\left(x_{k}\right)\right)\right) \bar{\psi}_{m}(x),
\end{aligned}
$$

further, $u_{1}(x) \in W_{1}[0,1]$ and $u_{1}(x)=\sum_{m=0}^{\infty}\left\langle u_{1}(x), \bar{\psi}_{m}(x)\right\rangle \bar{\psi}_{m}(x)$, the Fourier series expansion about orthonormal system $\left\{\bar{\psi}_{m}\right\}_{m=1}^{\infty}$ and $W_{1}[0,1]$ is a Hilbert space. Thus, the series $\sum_{m=0}^{\infty}\left\langle u_{1}(x), \bar{\psi}_{m}(x)\right\rangle \bar{\psi}_{m}(x)$ is convergent in the sense of $\|\cdot\|_{W_{1}}$. Similarly, for $u_{2}(x)$ in (4.5) we get

$$
u_{2}(x)=\sum_{m=1}^{\infty} \sum_{k=1}^{m} \beta_{m k}\left(f_{2}\left(x_{k}\right)+N_{2}\left(x_{k}, u_{1}\left(x_{k}\right)\right)+\widetilde{N}_{2}\left(x_{k}, u_{2}\left(x_{k}\right)\right)\right) \bar{\psi}_{m}(x) \text {. }
$$

By taking, a limited number of terms in the series solutions $u_{1}(x)$ and $u_{2}(x)$ to get the approximate solutions $u_{1 n}(x)$ and $u_{2 n}(x)$, respectively, as follows:

$$
\left.\begin{array}{l}
u_{1 n}(x)=\sum_{m=1}^{n} \sum_{k=1}^{m} \beta_{m k}\left(f_{1}\left(x_{k}\right)+N_{1}\left(x_{k}, u_{1}\left(x_{k}\right)\right)+\widetilde{N}_{1}\left(x_{k}, u_{2}\left(x_{k}\right)\right)\right) \bar{\psi}_{m}(x) \\
u_{2 n}(x)=\sum_{m=1}^{n} \sum_{k=1}^{m} \beta_{m k}\left(f_{2}\left(x_{k}\right)+N_{2}\left(x_{k}, u_{1}\left(x_{k}\right)\right)+\widetilde{N}_{2}\left(x_{k}, u_{2}\left(x_{k}\right)\right)\right) \bar{\psi}_{m}(x)
\end{array}\right\}
$$

Theorem 4.3 Consider $u_{1}(x)$ and $u_{2}(x) \in W_{1}[0,1]$ are the solutions of (4.1). Let $E_{1 n}(x)=u_{1}(x)-u_{1 n}(x)$ and $E_{2 n}(x)=u_{2}(x)-u_{2 n}(x)$. Then $E_{1 n}(x)$ and $E_{2 n}(x)$ are a monotone decreasing in the sense of the norm of $W_{1}[0,1]$. i.e., $E_{1 n} \rightarrow 0$ and $E_{2 n} \rightarrow 0$ as $n \rightarrow \infty$.

Proof. From $u_{1}(x)$ and $u_{1 n}(x)$ in (4.5) and (4.6) respectively.

$$
\begin{aligned}
& \left\|E_{1 n}\right\|_{W_{1}[0,1]}^{2}=\left\|u_{1}(x)-u_{1 n}(x)\right\|_{W_{1}[0,1]}^{2} \\
& =\left\|\sum_{m=n+1}^{\infty} \sum_{k=1}^{n} \beta_{m k}\left(f_{1}\left(x_{k}\right)+N_{1}\left(x_{k}, u_{1}\left(x_{k}\right)\right)+\widetilde{N}_{1}\left(x_{k}, u_{2}\left(x_{k}\right)\right)\right) \bar{\psi}_{m}(x)\right\|_{W_{1}[0,1]}^{2}=\left\|\sum_{m=n+1}^{\infty} A_{m} \bar{\psi}_{m}(x)\right\|_{W_{1}[0,1]}^{2}= \\
& \sum_{m=n+1}^{\infty}\left(A_{m}\right)^{2},
\end{aligned}
$$

and, $\quad\left\|E_{1 n-1}\right\|_{W_{1}[0,1]}^{2}=\sum_{m=n}^{\infty}\left(A_{m}\right)^{2}$.

Thus, $\left\|E_{1 n}\right\|_{W_{1}[0,1]}^{2} \leq\left\|E_{1 n-1}\right\|_{W_{1}[0,1]}^{2}$. Similarly from $u_{2}(x)$ and $u_{2 n}(x)$ in (4.5) and (4.6) respectively,

$\left\|E_{2 n}\right\|_{W_{1}[0,1]}^{2}=\left\|u_{2}(x)-u_{2 n}(x)\right\|_{W_{1}[0,1]}^{2}$

$=\left\|\sum_{m=n+1}^{\infty} \sum_{k=1}^{n} \beta_{m k}\left(f_{2}\left(x_{k}\right)+N_{2}\left(x_{k}, u_{1}\left(x_{k}\right)\right)+\widetilde{N}_{2}\left(x_{k}, u_{2}\left(x_{k}\right)\right)\right) \bar{\psi}_{m}(x)\right\|_{W_{1}[0,1]}^{2} \quad=\left\|\sum_{m=n+1}^{\infty} B_{m} \bar{\psi}_{m}(x)\right\|_{W_{1}[0,1]}^{2}=$ $\sum_{m=n+1}^{\infty}\left(B_{m}\right)^{2}$,

and $\quad\left\|E_{2 n-1}\right\|_{W_{1}[0,1]}^{2}=\sum_{m=n}^{\infty}\left(B_{m}\right)^{2}$.

Thus, $\quad\left\|E_{2 n}\right\|_{W_{1}[0,1]}^{2} \leq\left\|E_{2 n-1}\right\|_{W_{1}[0,1]}^{2}$. Where the coefficients $A_{m}, B_{m}$ of $\bar{\psi}_{m}(x), m=1, \ldots$.

Consequently, $E_{1 n}(x)$ and $E_{2 n}(x)$ are a monotone decreasing in the sense of the norm of $W_{1}[0,1]$.

Remark Since $W_{1}[0,1]$ is a Hilbert space, it is clear that $\sum_{m=n}^{\infty}\left(A_{m}\right)^{2}<\infty$ and $\sum_{m=n}^{\infty}\left(A_{m}\right)^{2}<\infty$. Therefore, the sequence $u_{1 n}$ and $u_{2 n}$ are convergent.

\section{5-Numerical Examples}


The RKHS method will be apply to solve two Examples. To illustrate the applicability and effectiveness of this method comparing with the exact solutions by find the absolute error (Abs. err.) between the exact and approximate solutions, by taking $x_{m}=\frac{m-1}{n-1}$, where $m=1, \ldots, n$, at some choices $n=50$ and100. Symbolic and numerical computations performed by using MATLAB17.

Example 5.1 [1] Consider the following system of linear weakly singular VIEs:

$$
\left.\begin{array}{l}
u_{1}(x)=f_{1}(x)+\int_{0}^{x}\left(\frac{3}{(x-y)^{\frac{1}{2}}} u_{1}(y)+\frac{2}{(x-y)^{\frac{1}{2}}} u_{2}(y)\right) d y \\
u_{2}(x)=f_{2}(x)+\int_{0}^{x}\left(\frac{2}{(x-y)^{\frac{1}{2}}} u_{1}(y)-\frac{3}{(x-y)^{\frac{1}{2}}} u_{2}(y)\right) d y,
\end{array}\right\} 0 \leq x \leq 1,
$$

The exact solution is $u_{1}(x)=x^{4}, u_{2}(x)=x^{3}$, let $f_{1}(x)=x^{4}-\frac{64}{105} x^{\frac{7}{2}}(4 x+3)$ and $f_{2}(x)=x^{3}-\frac{32}{315} x^{\frac{7}{2}}(16 x-$ 27). The numerical results are displayed in Tables 1 and 2.

Table 1. Results of $u_{1}(x)$ for Example 5. 1

\begin{tabular}{|c|c|c|c|c|c|}
\hline$x_{m}$ & Exact sol. & $\begin{array}{c}\text { Approximate } \\
\text { sol. } u_{1,50}(x)\end{array}$ & $\begin{array}{c}\text { Abs. err. } \\
u_{1,50}(x)\end{array}$ & $\begin{array}{c}\text { Approximate } \\
\text { sol. } u_{1,100}(x)\end{array}$ & $\begin{array}{c}\text { Abs. err. } \\
u_{1,100}(x)\end{array}$ \\
\hline 0.1 & $1.0000 e-04$ & $4.4407 e-05$ & $5.5593 e-05$ & $6.8301 e-05$ & $3.1699 e-05$ \\
\hline 0.2 & $1.6000 e-03$ & $1.1381 e-03$ & $4.6189 e-04$ & $1.3567 e-03$ & $2.4333 e-04$ \\
\hline 0.3 & $8.1000 e-03$ & $6.6639 e-03$ & $1.4361 e-03$ & $7.3629 e-03$ & $7.3706 e-04$ \\
\hline 0.4 & $2.5600 e-02$ & $2.2606 e-02$ & $2.9937 e-03$ & $2.4083 e-02$ & $1.5166 e-03$ \\
\hline 0.5 & $6.2500 e-02$ & $5.7552 e-02$ & $4.9480 e-03$ & $6.0013 e-02$ & $2.4872 e-03$ \\
\hline 0.6 & $1.2960 e-01$ & $1.2269 e-01$ & $6.9104 e-03$ & $1.2614 e-01$ & $3.4558 e-03$ \\
\hline 0.7 & $2.4010 e-01$ & $2.3181 e-01$ & $8.2904 e-03$ & $2.3597 e-01$ & $4.1307 e-03$ \\
\hline 0.8 & $4.0960 e-01$ & $4.0130 e-01$ & $8.2954 e-03$ & $4.0548 e-01$ & $4.1217 e-03$ \\
\hline 0.9 & $6.5610 e-01$ & $6.5017 e-01$ & $5.9308 e-03$ & $6.5316 e-01$ & $2.9405 e-03$ \\
\hline 1.0 & $1.0000 e+00$ & $1.0000 e+00$ & 0 & $1.0000 e+00$ & 0 \\
\hline
\end{tabular}




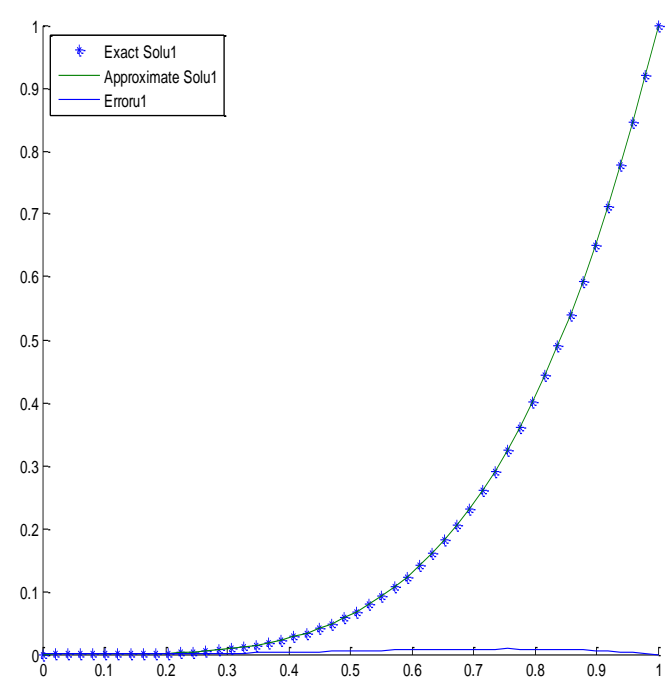

(a)

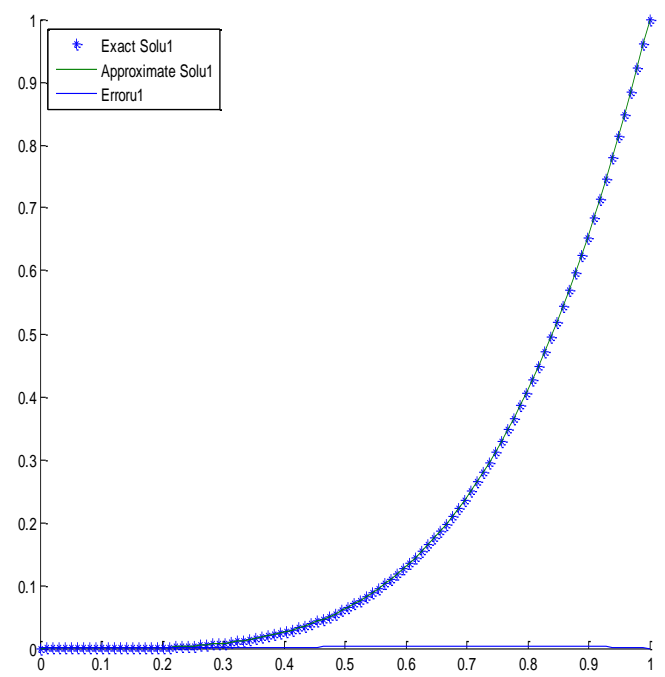

(b)

Figure 1: Solution of RKHS method for $u_{1}(x)$ for $n=50,100$ in (a) and (b) respectively.

Table 2. Results $u_{2}(x)$ for Example 5. 1

\begin{tabular}{|c|c|c|c|c|c|}
\hline$x_{m}$ & Exact sol. $u_{2}(x)$ & $\begin{array}{c}\text { Approximate } \\
\text { sol. } u_{2,50}(x)\end{array}$ & $\begin{array}{c}\text { Abs. err. } \\
u_{2,50}(x)\end{array}$ & $\begin{array}{c}\text { Approximate } \\
\text { sol. } u_{2,100}(x)\end{array}$ & $\begin{array}{c}\text { Abs. err. } \\
u_{2,100}(x)\end{array}$ \\
\hline 0.1 & $1.0000 e-03$ & $5.4399 e-04$ & $4.5601 e-04$ & $7.5131 e-04$ & $2.4869 e-04$ \\
\hline 0.2 & $8.0000 e-03$ & $6.1964 e-03$ & $1.8036 e-03$ & $7.0690 e-03$ & $9.3104 e-04$ \\
\hline 0.3 & $2.7000 e-02$ & $2.3324 e-02$ & $3.6764 e-03$ & $2.5136 e-02$ & $1.8644 e-03$ \\
\hline 0.4 & $6.4000 e-02$ & $5.8301 e-02$ & $5.6995 e-03$ & $6.1135 e-02$ & $2.8652 e-03$ \\
\hline 0.5 & $1.2500 e-01$ & $1.1750 e-01$ & $7.4979 e-03$ & $1.2125 e-01$ & $3.7497 e-03$ \\
\hline 0.6 & $2.1600 e-01$ & $2.0730 e-01$ & $8.6969 e-03$ & $2.1167 e-01$ & $4.3343 e-03$ \\
\hline 0.7 & $3.4300 e-01$ & $3.3408 e-01$ & $8.9215 e-03$ & $3.3856 e-01$ & $4.4353 e-03$ \\
\hline 0.8 & $5.1200 e-01$ & $5.0420 e-01$ & $7.7968 e-03$ & $5.0813 e-01$ & $3.8690 e-03$ \\
\hline 0.9 & $7.2900 e-01$ & $7.2405 e-01$ & $4.9479 e-03$ & $7.2655 e-01$ & $2.4518 e-03$ \\
\hline 1.0 & $1.0000 e+00$ & $1.0000 e+00$ & 0 & $1.0000 e+00$ & 0 \\
\hline
\end{tabular}




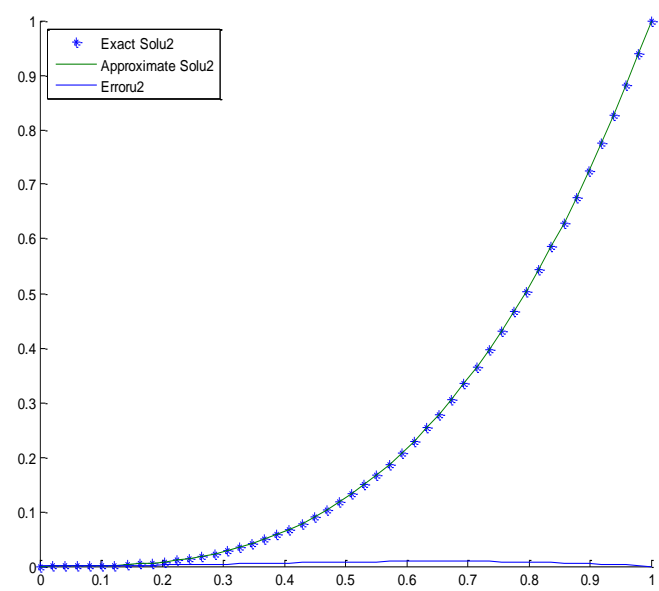

(a)

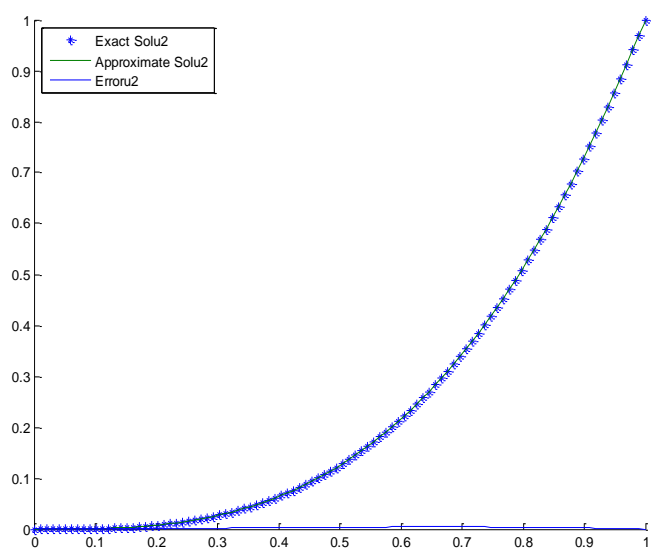

(b)

Figure 2: Solution of RKHS method for $u_{2}(x)$ for $n=50,100$ in (a) and (b) respectively.

Example 5.2 [1] Consider the following system of linear weakly singular VIEs:

$\left.\begin{array}{l}u_{1}(x)=f_{1}(x)+\int_{0}^{x}\left(\frac{1}{(x-y)^{\frac{1}{5}}} u_{1}(y)+\frac{1}{(x-y)^{\frac{2}{5}}} u_{2}(y)\right) d y \\ u_{2}(x)=f_{2}(x)+\int_{0}^{x}\left(\frac{1}{(x-y)^{\frac{3}{5}}} u_{1}(y)+\frac{1}{(x-y)^{\frac{4}{5}}} u_{2}(y)\right) d y,\end{array}\right\} 0 \leq x \leq 1$,

The exact solution is $u_{1}(x)=x+x^{2}, u_{2}(x)=x-x^{2}$, let $f_{1}(x)=x+x^{2}-\frac{25}{6552} x^{\frac{8}{5}}\left(130 x^{\frac{6}{5}}+182 x^{\frac{1}{5}}-210 x+\right.$ 273) and $f_{2}(x)=x-x^{2}-\frac{25}{924} x^{\frac{6}{5}}\left(55 x^{\frac{6}{5}}+66 x^{\frac{1}{5}}-140 x+154\right)$. The numerical results are displayed in Tables 3 and 4 .

Table 3. Results of $u_{1}(x)$ for Example 5. 2

\begin{tabular}{|c|c|c|c|c|c|}
\hline$x_{m}$ & Exact sol. & $\begin{array}{c}\text { Approximate } \\
\text { sol. } u_{1,50}(x)\end{array}$ & Abs. err. & $\begin{array}{c}\text { Approximate } \\
\text { sol. } u_{1,100}(x)\end{array}$ & Abs. err. \\
& $u_{1}(x)$ & $u_{1,50}(x)$ & & $u_{1,100}(x)$ \\
\hline 0.1 & $1.1000 e-01$ & $8.8297 e-02$ & $2.1703 e-02$ & $9.9174 e-02$ & $1.0826 e-02$ \\
\hline 0.2 & $2.4000 e-01$ & $2.1741 e-01$ & $2.2591 e-02$ & $2.2875 e-01$ & $1.1248 e-02$ \\
\hline 0.3 & $3.9000 e-01$ & $3.6735 e-01$ & $2.2653 e-02$ & $3.7874 e-01$ & $1.1263 e-02$ \\
\hline 0.4 & $5.6000 e-01$ & $5.3811 e-01$ & $2.1891 e-02$ & $5.4913 e-01$ & $1.0872 e-02$ \\
\hline 0.5 & $7.5000 e-01$ & $7.2970 e-01$ & $2.0304 e-02$ & $7.3992 e-01$ & $1.0076 e-02$ \\
\hline 0.6 & $9.6000 e+01$ & $9.4211 e-01$ & $1.7893 e-02$ & $9.5113 e-01$ & $8.8726 e-03$ \\
\hline 0.7 & $1.1900 e+00$ & $1.1753 e+00$ & $1.4656 e-02$ & $1.1827 e+00$ & $7.2635 e-03$ \\
\hline 0.8 & $1.4400 e+00$ & $1.4294 e+00$ & $1.0596 e-02$ & $1.4348 e+00$ & $5.2484 e-03$ \\
\hline 0.9 & $1.7100 e+00$ & $1.7043 e+00$ & $5.7101 e-03$ & $1.7072 e+00$ & $2.8273 e-03$ \\
\hline
\end{tabular}




\begin{tabular}{|l|c|c|c|c|c|}
\hline 1.0 & $2.0000 e+00$ & $2.0000 e+00$ & 0 & $2.0000 e+00$ & 0 \\
\hline
\end{tabular}

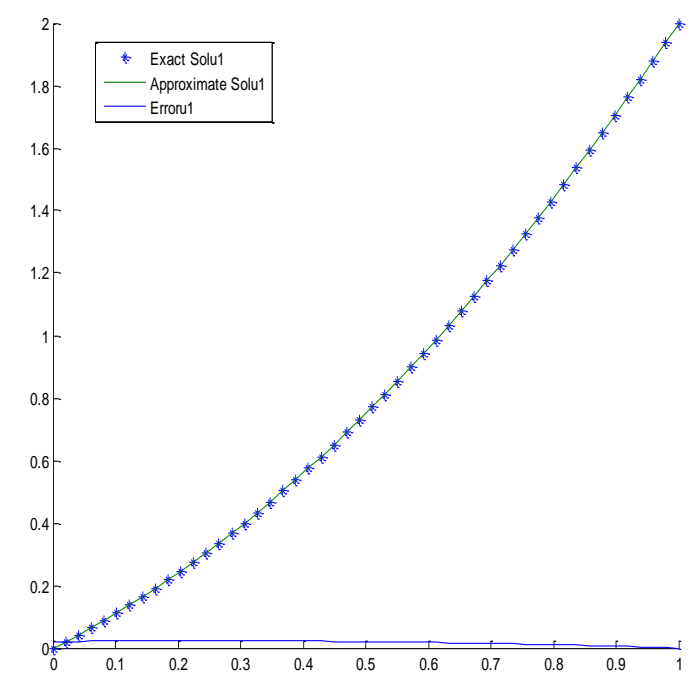

(a)

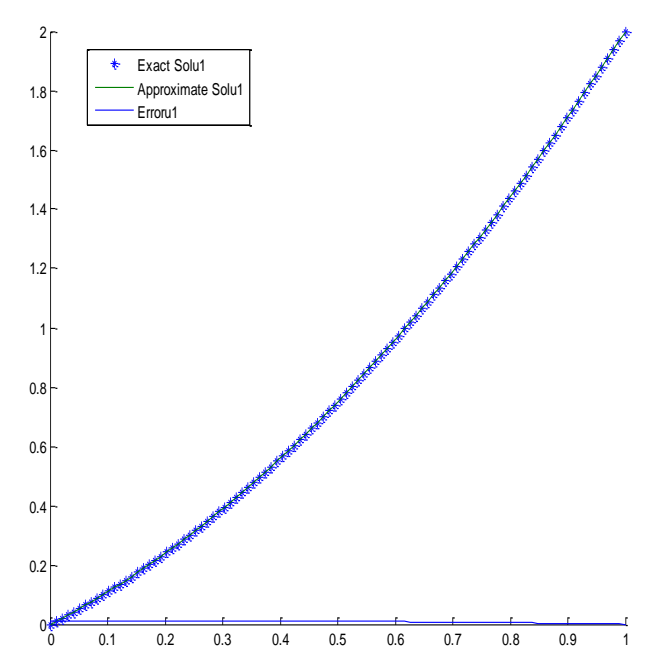

(b)

Figure 3: Solution of RKHS method for $u_{1}(x)$ for $n=50,100$ in (a) and (b) respectively.

Table 4. Results of $u_{2}(x)$ for Example 5. 2

\begin{tabular}{|c|c|c|c|c|c|}
\hline$x_{m}$ & Exact sol. & $\begin{array}{c}\text { Approximate } \\
\text { sol. } u_{2,50}(x)\end{array}$ & $\begin{array}{c}\text { Abs. err. } \\
u_{2,50}(x)\end{array}$ & $\begin{array}{c}\text { Approximate } \\
\text { sol. } u_{2,100}(x)\end{array}$ & $\begin{array}{c}\text { Abs. err. } \\
u_{2,100}(x)\end{array}$ \\
\hline 0.1 & $9.0000 e-02$ & $7.4969 e-02$ & $1.5031 e-02$ & $8.2645 e-02$ & $7.3554 e-03$ \\
\hline 0.2 & $1.6000 e-01$ & $1.4994 e-01$ & $1.0062 e-02$ & $1.5509 e-01$ & $4.9138 e-03$ \\
\hline 0.3 & $2.1000 e-01$ & $2.0408 e-01$ & $5.9184 e-03$ & $2.0712 e-01$ & $2.8783 e-03$ \\
\hline 0.4 & $2.4000 e-01$ & $2.3740 e-01$ & $2.5989 e-03$ & $2.3875 e-01$ & $1.2489 e-03$ \\
\hline 0.5 & $2.5000 e-01$ & $2.4990 e-01$ & $1.0412 e-04$ & $2.4997 e-01$ & $2.5508 e-05$ \\
\hline 0.6 & $2.4000 e-01$ & $2.4157 e-01$ & $1.5660 e-03$ & $2.4079 e-01$ & $7.9176 e-04$ \\
\hline 0.7 & $2.1000 e-01$ & $2.1241 e-01$ & $2.4115 e-03$ & $2.1120 e-01$ & $1.2029 e-03$ \\
\hline 0.8 & $1.6000 e-01$ & $1.6243 e-01$ & $2.4323 e-03$ & $1.6121 e-01$ & $1.2080 e-03$ \\
\hline 0.9 & $9.0000 e-02$ & $9.1628 e-02$ & $1.6285 e-03$ & $9.0807 e-02$ & $8.0706 e-04$ \\
\hline 1.0 & 0 & 0 & 0 & 0 & 0 \\
\hline
\end{tabular}




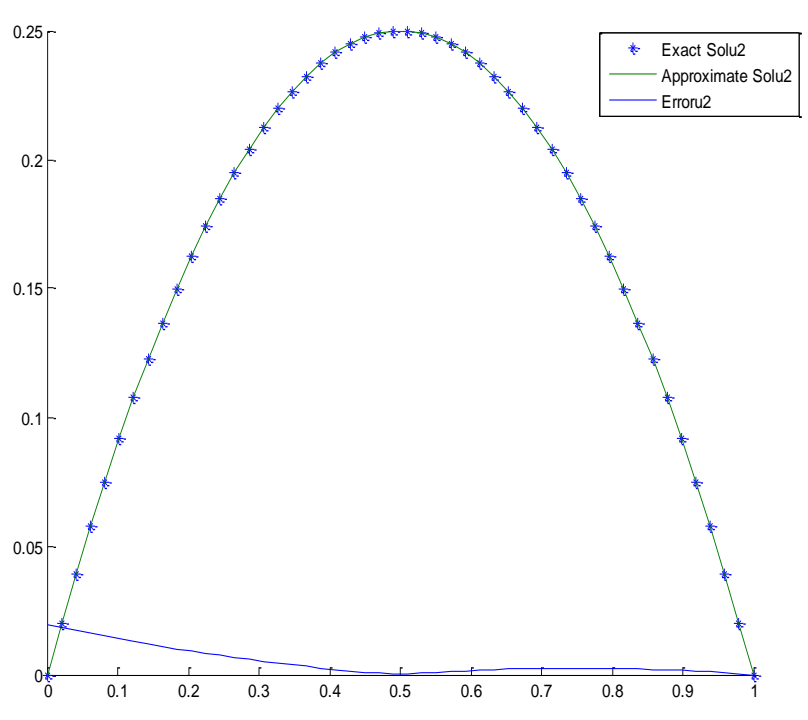

(a)

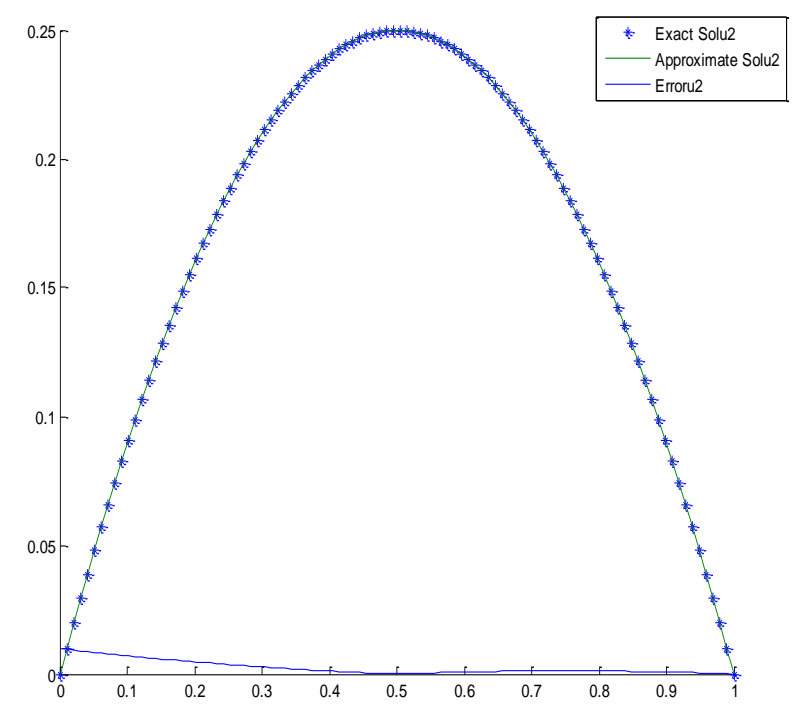

(b)

Figure 4: Solution of RKHS method for $u_{2}(x)$ for $n=50,100$ in (a) and (b) respectively.

\section{Conclusions}

In this paper, we have deduced the solution of system of linear weakly singular VIE by the solution system of LODE by using Taylor's expansion to get rid of the singularity. Then we have portray the solution of this system of LODE by RKHS method, which compared with the exact solution we found that the method is easy to apply and full efficiency in scientific applications to build a solution without linearization, turbulence or discretization. Therefore, it can concluded that the proposed method is very strong and powerful.

\section{References}

1. M. Wazwaz, Linear and nonlinear integral equations, Methods and Applications, HEP and Springer, Peking and Berlin, (2011).

2. Tahmasbi and O.S. Fard, Numerical solution of linear Volterra integral equations system of the second kind, Applied Mathematics and Computation, vol. 201. No. 1-2, pp.547-552, (2008).

3. E. Babolian, J. Biazar, and A. R. Vahidi, On the decomposition method for system of linear equations and system of linear Volterra integral equations, Applied Mathematics and Computation, vol. 147, no.1, pp. 1927, (2004).

4. J. Biazar and H. Ghazvini, He's homotopy perturbation method for solving systems of Volterra integral equations of the second kind, Chaos, Solutions and Fractals, vol. 39, no 2,pp. 770-777, (2009).

5. E. Yusufoglu, A homotopy perturbation algorithm to solve a system of Fredholm- Volterra type integral equations, Mathematical and Computer Modelling, vol.47, no. 11-12, pp. 1099-1107, (2008).

6.

R. Katani and S. Shahmorad, Block by block method for the systems of nonlinear Volterra integral equations, Applied Mathematical Modelling, vol. 34, no. 2, pp. 400-406,(2010). 
7. M. Rabbani, K. Maleknejad, and N. Aghazadeh, "Numerical computational solution of the Volterra integral equations system of the second kind by using an expansion method," Applied Mathematics and Computation, vol. 187, no. 2, pp. 1143-1146, (2007).

8. H. Yao, The research of algorithms for some singular differential equations of higher even-order, Department of mathematics, Harbin Institute of Technology, 2008.

9. M. Cui and Y. Lin, Nonlinear numerical Analysis in the Reproducing kernel space, Nova Science Publisher, New York, NY, USA, 2008.

10. A. Berlinet and C. Thomas-Agnan, Reproducing kernel Hilbert space in probability and statistics, Kluwer Academic Publishers, 2004.

11. Z. Chen and Y.Z. Lin, The exact solution of a linear integral equation with weakly Singular kernel, Journal of Mathematical Analysis and Applications, vol. 344, pp.726- 734, (2008).

12. S. Javadi, E. Babolian and E. Moradi, New implementation of reproducing kernel Hilbert space method for solving a class of functional integral equations, International Scientific Publications and consulting Services, vol. 2014, pp. 1-7, 2014. 